\title{
Fungitoxidade de Bidens pilosa, Thymus vulgaris, Lippia alba e Rosmarinus officinalis no desenvolvimento in vitro de fungos fitopatogênicos
}

\author{
Fungitoxicty of Bidens pilosa, Thymus vulgaris, \\ Lippia alba and Rosmarinus officinalis in the in vitro \\ development of phytophatogens fungi
}

\author{
Oscar Koutaro Tagami ${ }^{1}$; Marcos Diamantino G. Gasparin ${ }^{1}$; Kátia Regina Freitas \\ Schwan-Estrada ${ }^{1 *}$; Maria Eugênia da Silva Cruz ${ }^{1}$; Adriana Terumi Itako ${ }^{1}$; João \\ Batista Tolentino Júnior ${ }^{1}$; Luciano M. de Moraes ${ }^{1}$; José Renato Stangarlin²*
}

\section{Resumo}

\begin{abstract}
Avaliou-se in vitro, a atividade fungitóxica dos extratos brutos aquosos (EBA) em diferentes concentrações isolados e em misturas de Bidens pilosa, Thymus vulgaris, Lippia alba e Rosmarinus officinalis no desenvolvimento dos fungos Alternaria alternata, Colletotrichum graminicola, Rhizoctonia solani e Sclerotium rolfsii. A avaliação foi realizada incorporando os extratos brutos aquosos (EBAs) ao meio BDA isolados e em mistura, nas concentrações $0 \%, 1 \%, 5 \%, 10 \%, 15 \%, 25 \%$ e $50 \%$. Foram feitas medições diárias das colônias fúngicas até o momento em o tratamento controle cobriu $2 / 3$ da superfície do meio de cultura. Verificou-se que a mistura de T. vulgaris com B. pilosa não teve efeito positivo, pois o EBA isolado de $B$. pilosa não reduziu o crescimento do fungo $R$. solani e em mistura reduziu o efeito do EBA de T. vulgaris. Porém, o EBAs de R. officinalis e L. alba em mistura levaram a melhores resultados, reduzindo em até $60 \%$ o crescimento de A. alternata, do que os EBAs isoladamente. O extrato isolado de $T$. vulgaris reduziu em até $97 \%$ o crescimento de $C$. graminicola. Os resultados demonstraram que os extratos brutos das plantas testadas tiveram ação fungitóxica significativa contra os quatro fitopatógenos.
\end{abstract}

Palavras-chave: Plantas medicinais, Alternaria alternata, Colletotrichum graminicola, Rhizoctonia solani, Sclerotium rolfsii

\begin{abstract}
The fungitoxicity of aqueous crude extracts (ACE) of Bidens pilosa, Thymus vulgaris, Lippia alba and Rosmarinus officinalis was evaluated in vitro on development of Alternaria alternata, Colletotrichum graminicola, Rhizoctonia solani and Sclerotium rolfsii. The active ACE were incorporated to PotatoDextrose-Agar (PDA) alone or in mixture, at $0 \%, 1 \%, 5 \%, 10 \%, 15 \%, 25 \%$ and $50 \%$ concentrations. Measurement in fungal colonies was taken daily until the control treatment covered $2 / 3$ of the culture media. The mixtures of $T$. vulgaris with $B$. pilosa did not have positive effect because the ACE of $B$. pilosa did not reduce the fungal growth of $R$. solani and in mixture inhibited the effect ACE of T. vulgaris. However, the mixture of ACEs of $R$. officinalis and L. alba produced better results than
\end{abstract}

\footnotetext{
1 Universidade Estadual de Maringá, Departamento de Agronomia. Maringá-PR. E-mail: krfsestrada@uem.br; mescruz@wnet. com.br; atitako@yahoo.com.br; joaobtj@yahoo.com.br.

2 Universidade Estadual do Oeste do Paraná, Rua Pernambuco 1777, CEP 85960-000, Marechal Cândido Rondon, PR. Bolsista produtividade CNPq. E-mail: jrstangarlin@unioeste.br

* Autor para correspondência
} 
isolated ACEs, with $60 \%$ of growth inhibition of A. alternata. The ACE of T. vulgaris inhibited the growth of C. graminicola at $97 \%$. The results show that ACEs have significative fungitoxicity in the evaluated phythopathogens.

Key words: Medicinal plants, Alternaria alternata, Colletotrichum graminicola, Rhizoctonia solani, Sclerotium rolfsii

\section{Introdução}

Pesquisas desenvolvidas com extratos brutos ou óleos essenciais obtidos de plantas medicinais têm indicado o potencial das mesmas no controle de fitopatógenos, tanto por sua ação fungitóxica direta quanto pela indução de resistência, indicando a presença de compostos com características de eliciadores (SCHWAN-ESTRADA; STANGARLIN, 2005; STANGARLIN et al., 1999). Essa alternativa visa reduzir e/ou amenizar o uso de produtos químicos, haja visto que o seu uso intensivo na agricultura acarreta uma série de problemas ambientais como a contaminação dos alimentos, solo e água, intoxicação dos agricultores/ aplicadores, resistência de fitopatógenos e eliminação de microrganismos benéficos do solo (MOREIRA, 1996; PRITHIVIRAJ et al., 1997; STADNIK; TALAMINI, 2004). Na literatura é possível encontrar um grande número de trabalhos que citam as propriedades antimicrobianas dos compostos secundários de plantas medicinais para o controle de fitopatógenos. Nesses trabalhos, observam-se efeitos fungitóxicos em experimentos realizados in vitro e in vivo, utilizando extratos brutos ou os óleos essenciais de plantas medicinais. Como exemplos de trabalhos in vitro, têm-se os resultados obtidos com extrato de alho (Allium sativum) em Pythium ultimum, Colletotrichum lindemuthianum e Rhizoctonia solani (BIANCHI et al., 1997); em Phytophthora infestans (KEQIANG; BRUGGEN, 2001); extrato de arruda (Ruta graveolens), carqueja (Baccharis trimera) e alfavaca (Ocimum basilicum) em Sclerotinia rofsii, Alternaria alternata, $R$. solani, Phytophthora sp. e C. graminicola (STANGARLIN et al., 1999) e de genbibre (Zingiber officinalis) e Corymbia citriodora (eucalipto) em Helminthosporium sp. (RODRIGUES et al., 2006). Os óleos essenciais de Thymus vulgaris, Lavandula sp. e Mentha piperita em $C$. lindemuthianum e $P$. ultimum (ZAMBONELLI et al., 1996); óleos essenciais de C. citratus, E. citriodora e Ageraturm conizoides em Dydimella bryoniae (FIORI et al., 2000); e de Corymbia citriodora, Cymbopogon nardus, Azadirachta indica e T. vulgaris em urediniósporos de Phakopsora pachyrhizi (MEDICE et al., 2007). Já os exemplos in vivo, têm-se o controle da requeima (Phytophthora infestans) em batata pelo extrato de cavalinha (Equisetum sp.) (KE-QIANG; BRUGGEN, 2001); dooídio(Oidium lycopersici) em tomateiro por extratos e óleo de Azadirachta indica (CARNEIRO, 2003), da mancha marrom (Bipolaris sorokiniana) em trigo usando extrato aquoso de Artemisia camphorata (cânfora) (FRANZENER et al., 2003), e da pinta preta (Alternaria solani) em tomateiro por extrato de cúrcuma (Curcuma longa) e curcumina (componente do óleo essencial de $C$. longa) (BALBI-PEÑA et al., 2006a, 2006b).

Visto que a utilização de extratos e óleos oriundos de plantas medicinais tem mostrado resultados promissores no controle de patógenos de plantas, o presente trabalho teve como objetivo verificaro efeito dos extratos brutos aquosos de Bidens pilosa (picãopreto), Thymus vulgaris (tomilho), Lippia alba (erva cidreira) e Rosmarinus officinalis (alecrim) isolados e em mistura no crescimento micelial dos fungos Alternaria alternata, Colletotrichum graminicola, Rhizoctonia solani e Sclerotium rolfsii.

\section{Material e métodos}

Obtenção dos isolados

Os isolados de Alternaria alternata, Colletotrichum graminicola, Rhizoctonia solani e Sclerotium rolfsii foram cedidos pela micoteca do Laboratório de Fisiologia do Parasitismo da ESALQ/USP. Os mesmos foram mantidos em meio 
de cultura BDA (batata-dextrose-ágar), em câmara de crescimento, a uma temperatura de $25^{\circ} \mathrm{C} \pm 2{ }^{\circ} \mathrm{C}$ com luz fluorescente contínua.

\section{Extrato bruto aquoso sobre o crescimento micelial dos fungos}

Folhas frescas de B. pilosa (picão preto), $T$. vulgaris (tomilho), L. alba (erva cidreira) e $R$. officinalis (alecrim) foram coletadas e lavadas em água destilada. Depois, foram trituradas em caldo de batata $(20 \%)$ por $3 \mathrm{~min}$. no liquidificador. Os homogenatos resultantes foram filtrados em gaze e em papel de filtro Whatman nำ1, obtendo-se o extrato aquoso bruto (EBA). Este foi incorporado ao BDA (batata-dextrose-ágar) de maneira a se obter concentrações de $1 \%, 5 \%, 10 \%, 20 \%, 25 \%$ e $50 \%$, esterilizado por autoclavagem e distribuído em placas de Petri. Após a solidificação do meio, disco de micélio ( $8 \mathrm{~mm}$ de diâmetro) dos isolados com 10 dias de idade em BDA, foi repicado para o centro das placas, as quais foram vedadas com filme plástico e mantidas a $25^{\circ} \mathrm{C} \pm 2^{\circ} \mathrm{C}$ no escuro. No tratamento controle, foram utilizadas placas contendo somente BDA. A avaliação foi realizada através da medição diária do diâmetro das colônias, pela média de duas medidas diametralmente opostas, iniciando 24 horas após a instalação do experimento e perdurando até o momento em que as colônias do tratamento controle cobrissem $2 / 3$ da superfície do meio de cultura. Para o cálculo dos porcentuais de inibição do crescimento (PIC) micelial em meio de cultivo utilizou-se à fórmula (BASTOS, 1997): $\mathrm{PIC}=($ cresc. Testemunha - cresc. Tratamento/cresc . Testemunha) x 100 .

Efeito da mistura dos extratos brutos aquosos (EBA)

Para avaliar o efeito da mistura do extrato bruto de B. pilosa com T. vulgaris e R. officinalis com L. alba, folhas frescas de ambas as plantas foram trituradas em liquidificador, em água destilada por 3 min. Os homogenatos resultantes foram filtrados em gaze e incorporados ao BDA nas mesmas proporções de $1 \%, 5 \%, 10 \%, 15 \%, 20 \%, 25 \%$ e $50 \%$. Para a avaliação do crescimento micelial dos fitopatógenos foi utilizada a mesma metodologia descrita acima.

Utilizou-se o delineamento inteiramente casualizado (DIC) em esquema fatorial 4x6x8, com 4 repetições, cujos fatores foram 4 fungos, 6 EBAS (4 isolados e 2 misturas) e 8 concentrações.

\section{Resultados e discussão}

Em relação aos dados de crescimento micelial, pela análise de regressão, ajustou-se uma equação quadrática para todos os tratamentos. $\mathrm{O}$ fungo $A$. alternata (Figura 1A) teve seu crescimento reduzido em $12,9 \%$ na concentração de $27,3 \%$ do EBA de $B$. pilosa, e para o EBA de T. vulgaris a redução foi de $31,2 \%$ na concentração de $35,1 \%$. A mistura de B. pilosa e T. vulgaris, não proporcionou redução, pois somente na concentração de $41,1 \%$ a redução foi de apenas 22,6\%. A mistura de $L$. alba com $R$. officinalis (Figura 1B) proporcionou maior redução, pois na concentração de $33,4 \%$ a redução foi de $60 \%$, sendo que para o EBA isolado de L. alba a redução foi de 13,1\% para uma concentração de $38,9 \%$ e para o EBA de $R$. officinalis a redução foi de $56,1 \%$ para uma concentração de $33,1 \%$.

A mistura dos EBAs de B. pilosa com T. vulgaris proporcionou maior redução no crescimento de $C$. graminicola (Figura 2A), pois somente com 26\% da concentração da mistura o crescimento foi reduzido em 35,9\%. O EBA isolado de B. pilosa na concentração de $31,2 \%$ reduziu somente $27 \%$ e o EBA de T. vulgaris reduziu $37,3 \%$ na maior concentração, de $50 \%$ do extrato. Já os EBAs isolados de L. alba e R. officinalis (Figura 2B) reduziram significativamente o crescimento em até $97 \%$ nas concentrações de $36,4 \%$ e $39,1 \%$, respectivamente. A mistura desses EBAs também reduziu significativamente o crescimento, porém a redução foi de $91 \%$ em concentrações próximas dos EBAs isolados. 


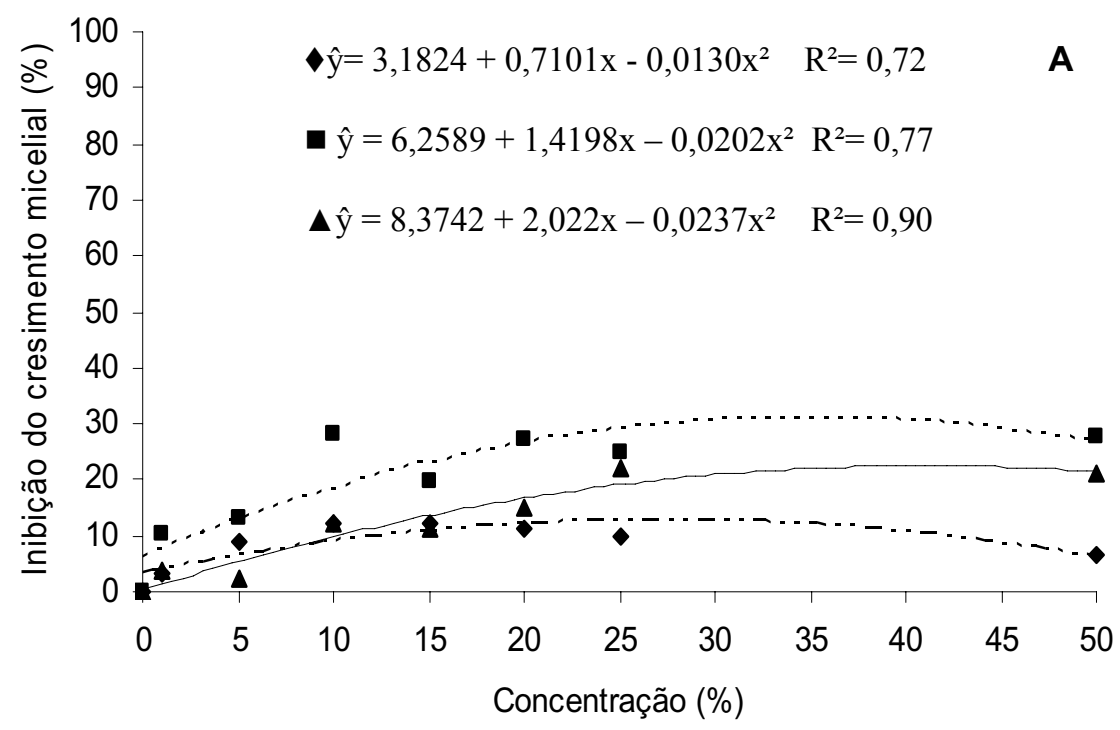

- Bidens pilosa - Thymus vulgaris \ Bidens pilosa + Thymus vulgaris

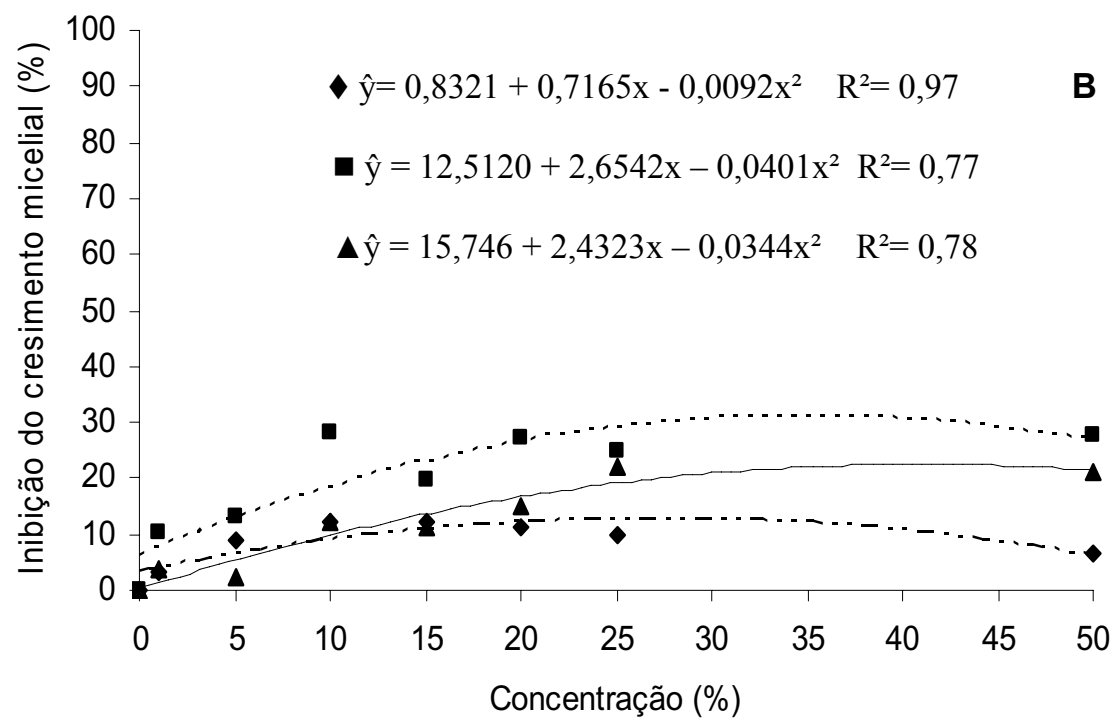

- Bidens pilosa - Thymus vulgaris \Bidens pilosa + Thymus vulgaris

Figura 1. Inibição do crescimento micelial (\%) de Alternaria alternata sob os tratamentos dos extratos brutos aquosos de Bidens pilosa, Thymus vulgaris, e Bidens pilosa + Thymus vulgaris (A); Lippia alba, Rosmarinus officinalis e Lippia alba + Rosmarinus officinalis (B) nas concentrações de 0, 1, 5, 10, 15, 20, 25 e 50\%. 


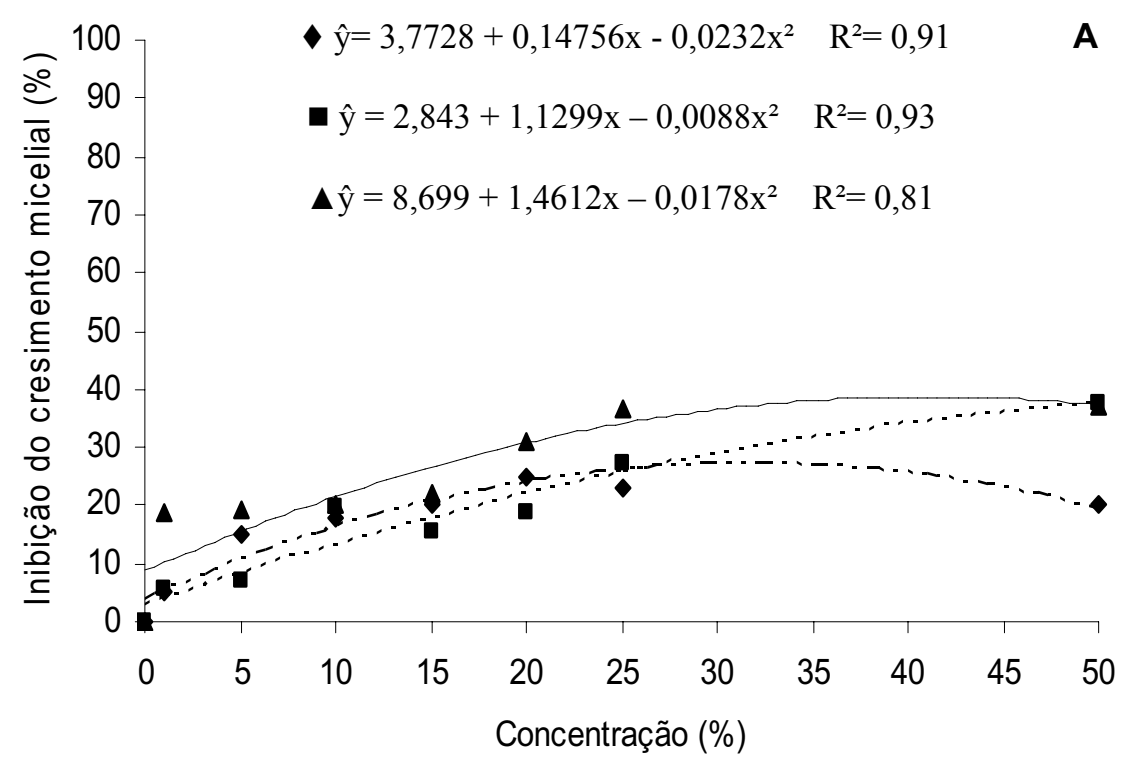

- Bidens pilosa — Thymus vulgaris \Bidens pilosa + Thymus vulgaris

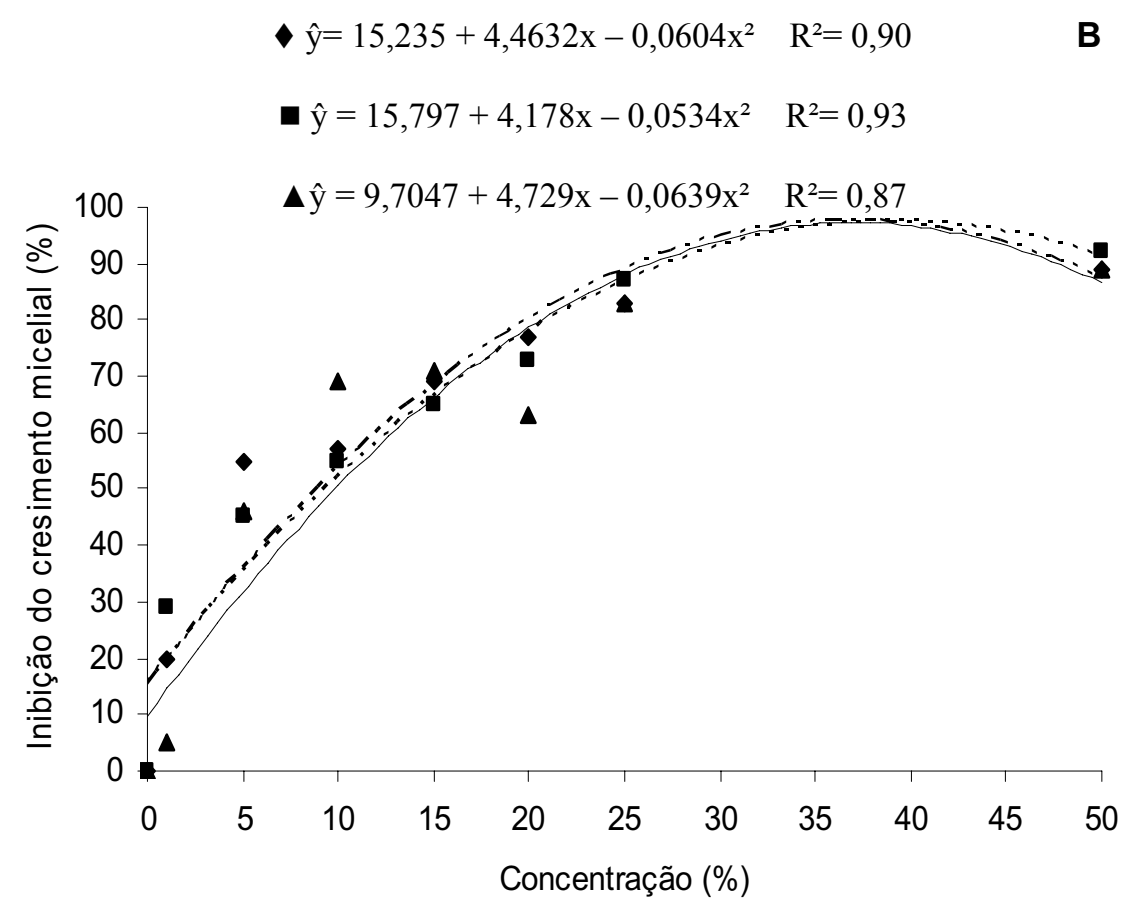

\section{- Lippia alba - Rosmarinus officinalis \ Lippia alba + Rosmarinus officinalis}

Figura 2. Inibição do crescimento micelial de Colletotrichum graminicola sob os tratamentos dos extratos brutos aquosos de Bidens pilosa, Thymus vulgaris, e Bidens pilosa + Thymus vulgaris (A); Lippia alba, Rosmarinus officinalis e Lippia alba + Rosmarinus officinalis (B) nas concentrações de 0, 1, 5, 10, 15, 20, 25 e 50\%. 
O EBA de $B$. pilosa não reduziu o crescimento do fungo $R$. solani em nenhuma das concentrações (Figura 3A) e a mistura reduziu em 37,2\% do crescimento na concentração de $38,4 \%$ e o EBA de T. vulgares isoladamente reduziu em até $55,6 \%$ o crescimento do fungo, quase na maior concentração do EBA testada. O EBA de $R$. officinalis (Figura 3B) reduziu significativamente o crescimento em $71,9 \%$ na concentração de $42,5 \%$, já o EBA de $R$. officinalis reduziu o crescimento em até $55,6 \%$. A mistura não proporcionou maior redução, pois reduziu $37 \%$ do crescimento na concentração de $38,4 \%$.

Já o crescimento de $S$. rolfsii (Figura 4A) teve reduções de crescimento micelial semelhantes com o EBA de T. vulgaris isolado e em mistura, próximos de $52 \%$ nas concentrações próximas a $39 \%$. Para o EBA isolado de B. pilosa, a redução foi pequena em concentrações próximas de $40 \%$. O mesmo ocorreu para o EBA isolado e em mistura de $R$. officinalis (Figura 4B), porém as reduções foram significativamente altas, em torno de $92 \%$, na concentração de 39,6\%. Para o EBA isolado de $L$. alba a maior redução ocorreu na maior concentração do extrato, com uma redução de $49 \%$.

Com estes resultados, pode-se verificar que em casos de mistura, como o de T. vulgaris com $B$. pilosa, os efeitos não foram positivos, pois o EBA isolado de $B$. pilosa não reduziu o crescimento do fungo $R$. solani e em mistura reduziu o efeito do EBA de T. vulgaris, que isolado teve efeito positivo. Porém, o EBAs de R. officinalis e L. alba em mistura levaram a melhores resultados do que isoladamente no crescimento micelial de $A$. alternata. Resultados semelhantes foram verificados por Baldo et al. (2005) que avaliaram a fungitoxidade in vitro dos extratos brutos de C. citratus e Cymbopogon nardus sobre Cladosporium fulvum isoladamente e em mistura, nas concentrações de $1 \%, 5 \%, 10 \%, 15 \%$ e $20 \%$. Nesse trabalho, a atividade antifúngica foi presente em todos os parâmetros avaliados, sendo a média de inibição de $77 \%$ para a massa micelial, $71 \%$ para esporulação e $24 \%$ para germinação de esporos, enquanto que, para o fungicida azoxystrobin $(4,0 \mathrm{~g}$ i.a/L), utilizado como controle positivo, as inibições foram de $91 \%, 79 \%$ e $76 \%$, respectivamente.

Bonaldo, Cruz e Schwan-Estrada (1999), quando trabalharam com C. citratus e E. citriodora, constataram que as concentrações de $25 \%$ e $50 \%$ do extrato de E. citriodora inibiram o crescimento micelial de Alternaria stevia em $35 \%$ e $40 \%$, e em $51 \%$ e $62 \%$ para $A$. solani. O extrato de $C$. citratus nas mesmas concentrações inibiu o crescimento de A. stevia em $29 \%$ e $30 \%$, e em $A$. solani a inibição foi em torno de $12 \%$ paras as concentrações acima de $15 \%$ do extrato bruto. Quando avaliaram o efeito da mistura dos extratos observaram uma inibição de $17 \%$ e $41 \%$ no crescimento de $A$. stevia e $A$. solani, respectivamente.

Vários outros trabalhos tiveram resultados positivos com o uso de extratos de plantas medicinais no desenvolvimento de fitopatógenos, como o de Valarini, Frighetto e Melo (1994), onde o extrato de $C$. citratus a $10 \%$ inibiu completamente o crescimento in vitro de vários patógenos causadores de podridão radiculares em feijão. Fiori et al. (2000), verificaram que os extratos brutos de Eucalyptus citriodora, Ageratum conyzoides e Achillea millefollium, além de inibirem o crescimento micelial, inibiram a germinação dos esporos de Didymella brioniae. Franzener et al. (2003), cujo extrato aquoso de cânfora a $50 \%$ causou a inibição de $39 \%$ do crescimento micelial de $B$. sorokiniana, sendo que a $10 \%$ já inibiu completamente a esporulação.

Rodrigues et al. (2006) mostraram também a eficiência dos extratos de Zingiber officinalis e Corymbia citriodora na redução do crescimento micelial e produção de esporos de Helminthosporium sp. O trabalho de Balbi-Peña et al. (2006a) indicou que os extratos de cúrcuma (Curcuma longa) a $10 \%$ e $15 \%$ não autoclavados inibiram em $38,2 \%$ e $23,2 \%$, respectivamente, o crescimento micelial e $71,7 \%$ e $87 \%$, respectivamente, a esporulação do fungo. 


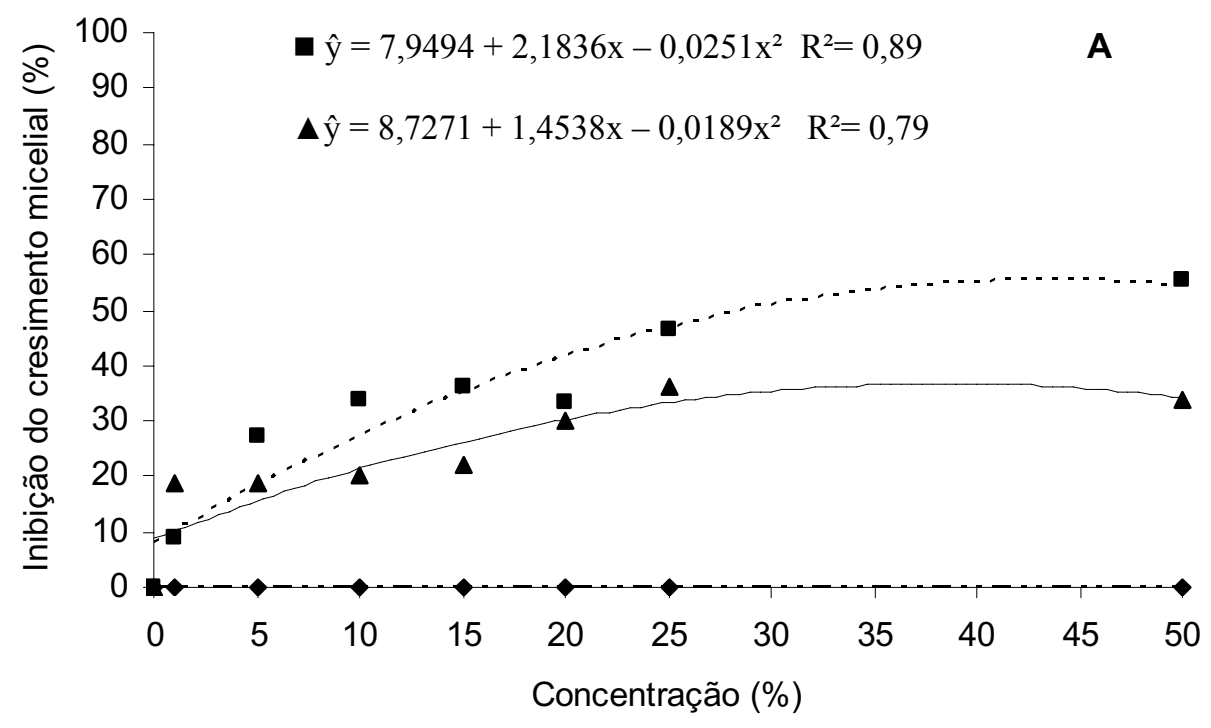

- Bidens pilosa - Thymus vulgaris \Bidens pilosa + Thymus vulgaris

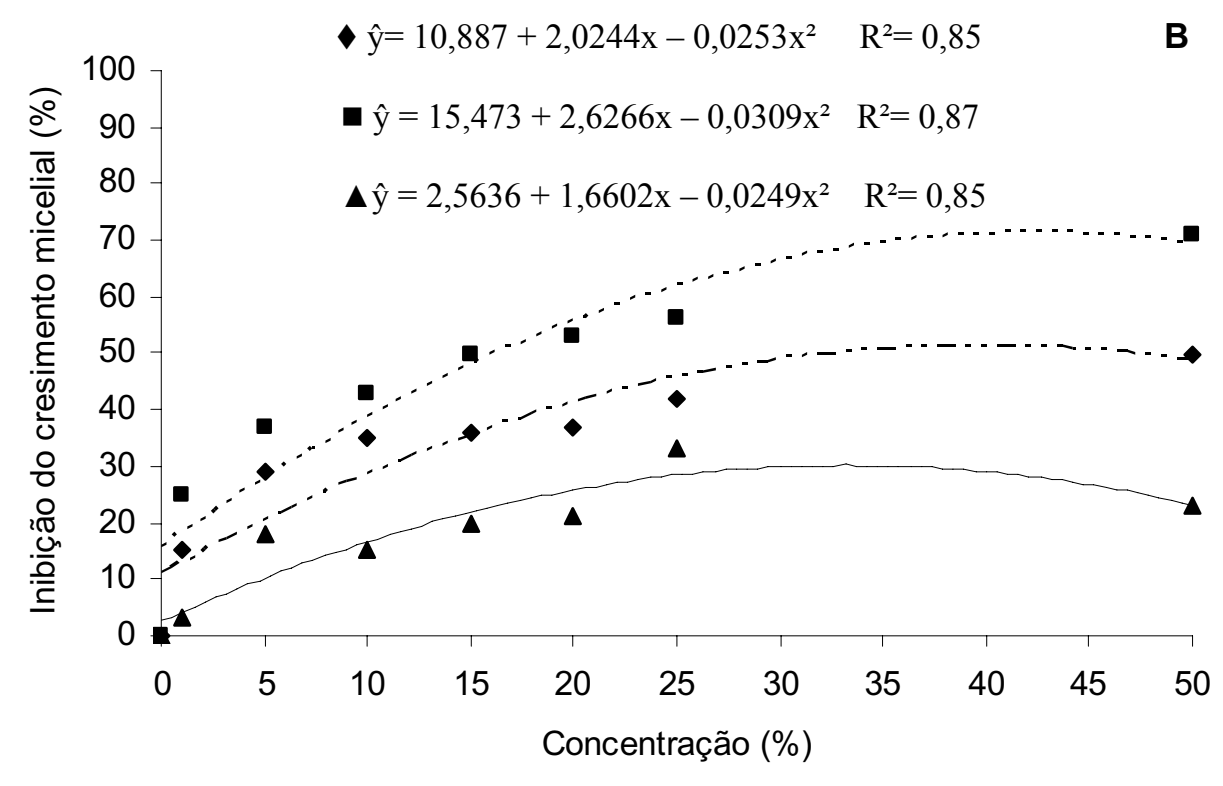

- Lippia alba - Rosmarinus officinalis \ Lippia alba + Rosmarinus officinalis

Figura 3. Inibição do crescimento micelial de Rhizoctonia solani sob os tratamentos dos extratos brutos aquosos de Bidens pilosa, Thymus vulgaris, e Bidens pilosa + Thymus vulgaris (A); Lippia alba, Rosmarinus officinalis e Lippia alba + Rosmarinus officinalis (B) nas concentrações de $0,1,5,10,15,20,25$ e $50 \%$. 


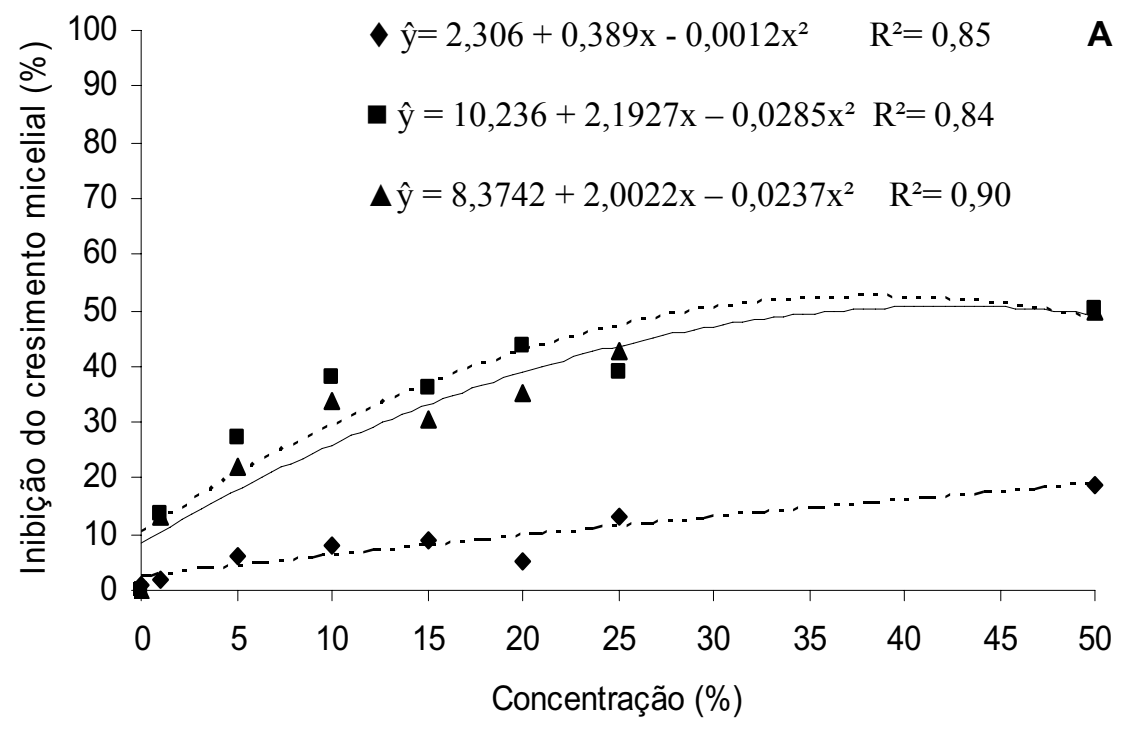

- Bidens pilosa - Thymus vulgaris \Bidens pilosa + Thymus vulgaris

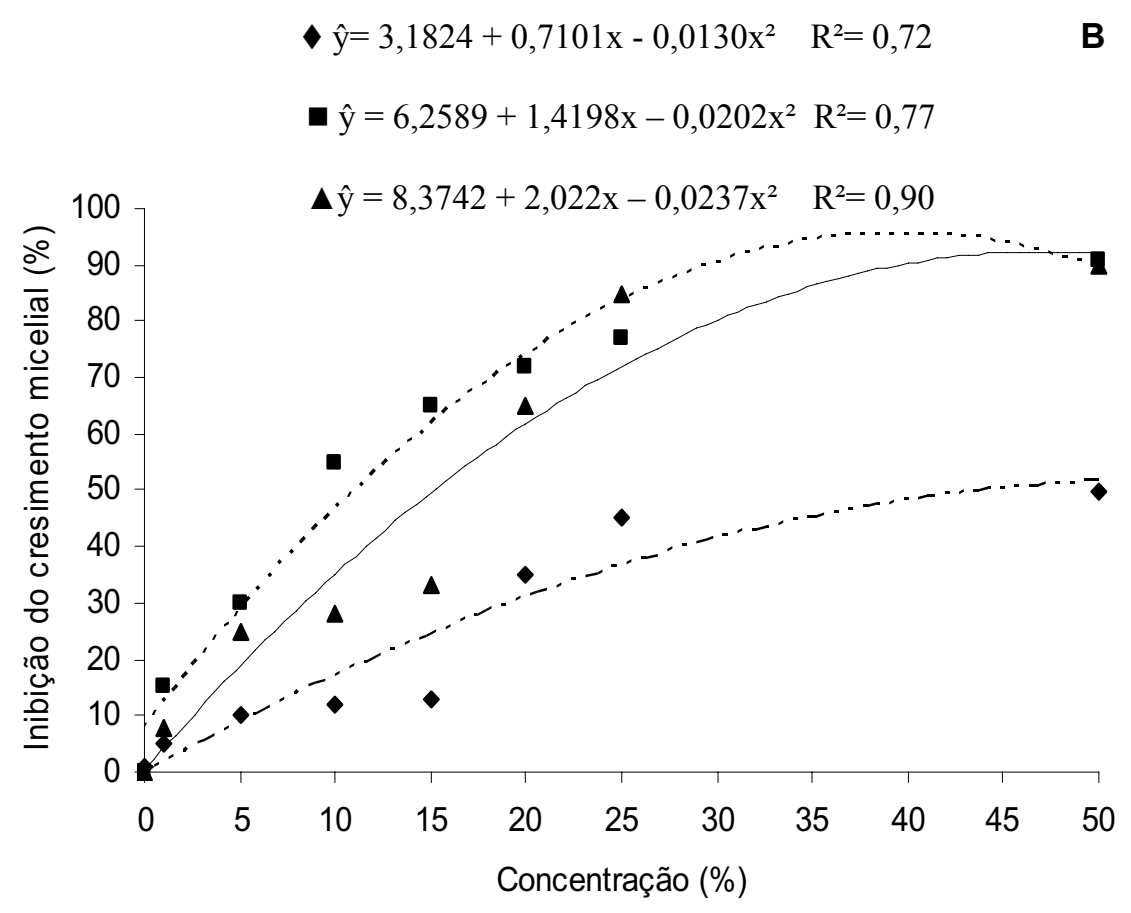

- Lippia alba - Rosmarinus officinalis ^ Lippia alba + Rosmarinus officinalis

Figura 4. Inibição do crescimento micelial de Sclerotinia rolfsii sob os tratamentos dos extratos brutos aquosos de Bidens pilosa, Thymus vulgaris, e Bidens pilosa + Thymus vulgaris (A); Lippia alba, Rosmarinus officinalis e Lippia alba + Rosmarinus officinalis (B) nas concentrações de $0,1,5,10,15,20,25$ e $50 \%$. 
Os resultados obtidos no presente trabalho demonstraram que os extratos brutos das plantas testadas tiveram ação fungitóxica significativa contra os quatros fitopatógenos, indicando, portanto, boas perspectivas para uso experimental desses extratos, tanto isolados como em mistura no controle desses fitopatógenos em condições de casa-de-vegetação e de campo. A possibilidade de uso de produtos de origem natural, que apresentam baixa toxicidade, é uma vantagem por ser um procedimento menos agressivo ao meio ambiente e à saúde humana.

\section{Conclusões}

Os extratos brutos das plantas medicinais testadas possuem ação fungitóxica in vitro sobre o crescimento micelial dos fungos Alternaria alternata, Colletotrichum graminicola, Rhizoctonia solani e Sclerotium rolfsii.

A mistura dos extratos não causou efeito positivo na inibição do crescimento micelial.

\section{Referências}

BALBI-PEÑA, M. I.; BECKER, A.; STANGARLIN, J. R.; FRANZENER, G.; LOPES M. C.; SCHWANESTRADA, K. R. F. Controle de Alternaria solani em tomateiro por extratos de Curcuma longa e curcumina I. avaliação in vitro. Fitopatologia Brasileira, Brasília, v. 31, n. 3, p. 310-314, 2006a.

Controle de Alternaria solani em tomateiro por extratos de Curcuma longa e curcumina - II. Avaliação in vivo. Fitopatologia Brasileira, Brasília, v. 31, n. 4, p. 401-404 2006b.

BALDO, M.; SORNBERGER, A.; STANGARLIN, J. R.; GRISA, S.; ECKSTEIN, B.; GIESE, C.; SCHWANESTRADA, K. R. F. Potencial do extrato bruto de Cymbopogon citratus (capim-limão) e Cymbopogon nardus (citronela) no controle in vitro de Cladosporium fulvum do tomateiro. In: JORNADA CIENTÍFICA DA UNIOESTE, 3., 2005, Marechal Cândido Rondon. Anais... Marechal Cândido Rondon: PRPPG - Unioeste, 2005. p. 1-5.

BASTOS, C.N. Efeito do óleo de Piper aduncum sobre Crinipelis e outros fungos fitopatogênicos. Fitopatologia Brasileira, Brasília, v. 22, n. 3, p.441-443, 1997.
BIANCHI, A.; ZAMBONELLI, A.; D’AURELIO, A. Z.; BALLESIA, F. Ultrastructural studies of the effects of Allium sativum on phytopathogenic fungi in vitro. Plant Disease, Saint Paul, v. 81, n. 11, p. 1241-1246, 1997.

BONALDO, S. M.; CRUZ, M. E. S.; SCHWANESTRADA, K. R. F. Potencial das ervas medicinais capimlimão (Cymbopogon citratus) e eucalipto (Eucalyptus citriodora) no controle de fungos fitopatogênicos. In: CONGRESSO PAULISTA DE FITOPATOLOGIA, 22., Jaboticabal, 1999. Resumos... Jaboticabal : GPF, 1999. p. 111.

CARNEIRO, S. M. T. P. G. Efeito de extratos de folhas e do óleo de nim sobre o oídio do tomateiro. Summa Phytopathologica, Botucatu, v. 29, n. 3, p. 262-265, 2003.

FIORI,A.C. G.; SCHWAN-ESTRADA, K. R. F.; STANGARLIN, J. R.; VIDA,J. B.; SCAPIM, C. A, CRUZ, M. E. S.; PASCHOLATI, S. F. Antifungal activity of leaf extracts and essential oils of some medicine plants against Didymella bryoniae. Journal of Phytopathology, Berlin, v. 148, n. 7-8, p. 493-487, 2000.

FRANZENER, G.; STANGARLIN, J. R.; SCHWANESTRADA, K. R. F.; CRUZ, M. S. Atividade antifúngica e indução de resistência em trigo a Bipolaris sorokiniana por Artemisia camphorata. Acta Scientiarum, Maringá, v. 25, n. 2, p. 503-507, 2003.

KE-QIANG, C.; BRUGGEN, A. H. C. Inhibitory efficacy of several plant extracts and plant products on Phytophthora infestans. Journal of Agricultural University of Hebei, Baoding, v. 24; n. 12, p. 108-116, 2001.

MEDICE, R.; ALVES, E.; ASSIS, R. T.; MAGNOJÚNIOR, R. G.; LOPES, E. A. G. L. Óleos essenciais no controle da ferrugem asiática da soja Phakopsora pachyrhizi Syd. \& P. Syd. Ciência Agrotecnologia, Lavras, v. 31, n. 1, p. 83-90, 2007.

MOREIRA, F. Plantas que curam. 5. ed. São Paulo: Hemus, 1996.

PRITHIVIRAJ, B.; SINGH, U. P.; MANICKAM, M.; SRIVASTAVA, J. S.; RAY, A. B. Antifungal activity of bergenin, a constituent of Flueggea microcarpa. Plant Pathology, Oxford, v. 46, n. 2, p. 224-228, 1997.

RODRIGUES, E.; SCHWAN-ESTRADA, K. R. F.; STANGARLIN, J. R.; CRUZ, M. E. S, TUTIDA-FIORI, A. C. G. Avaliação da atividade antifúngica de extratos de gengibre e eucalipto in vitro e em fibras de bananeira infectadas com Helminthosporium sp. Acta Scientiarum Agronomy, Maringá, v. 28, n. 1, p. 123-127, 2006. 
SCHWAN-ESTRADA, K. R. F; STANGARLIN, J. R. Extratos e óleos essenciais de plantas medicinais na indução de resistência. In: CAVALCANTI, L. S.; DI PIERO, R. M.; CIA, P.; PASCHOATI, S. F.; RESENDE, M. L. V.; ROMEIRO, R. S. Indução de resistência em plantas a patógenos e insetos. Piracicaba: Fealq, 2005. p. 125-132.

STADNIK, M. J.; TALAMINI, V. Manejo ecológico de doenças de plantas. Florianópolis: Ed. da UFSC, 2004.

STANGARLIN, J. R.; SCHWAN-ESTRADA, K. R. F; CRUZ, M. E. S., NOZAKI, M. H. Plantas medicinais e controle alternativo de fitopatógenos. Biotecnologia Ciência \& Desenvolvimento, Brasília, v. 2, n. 11, p. 1621, 1999.

VALARINI, P. J.; FRIGHETTO, R. T. S.; MELO, I. S. Potencial da erva medicinal (Cymbopogon citratus) no controle de fitopatógenos do feijoeiro. Revista de Agricultura, Piracicaba, v. 69, n. 4, p. 139-150, 1994.

ZAMBONELLI, A.; D'AURELIO, A. Z.; BIANCHI, A.; ALBASIN, A. Effects of essential oils on phytopathogenic fungi in vitro. Journal of Phytopathology, Berlin, v. 144, n. 3, p. 491-494. 1996. 